\title{
Advances in Momentum Resolved EELS
}

Benjamin Plotkin-Swing ${ }^{1}$, George Corbin ${ }^{2}$, Niklas Dellby ${ }^{1}$, Nils Johnson ${ }^{3}$, Petr Hrncrik ${ }^{3}$, Chris Meyer ${ }^{4}$, Andreas Mittelberger ${ }^{1}$, Dylan Taylor ${ }^{3}$, Ondrej Krivanek ${ }^{1}$ and Tracy Lovejoy ${ }^{1}$

${ }^{1}$ Nion R\&D, 11511 NE 118th St, Kirkland, WA, 98034, USA, United States, ${ }^{2}$ Nion Co., Kirkland, Washington, United States, ${ }^{3}$ Nion Co., United States, ${ }^{4}$ Nion R\&D, 11511 NE 118th St, Kirkland, WA, 98034, USA, Washington, United States

Vibrational modes affect conduction of heat and sound in solids, and are altered by local structure such as defects and interfaces. Angle Resolved Electron Energy Loss Spectroscopy (AR-EELS) within the Scanning Transmission Electron Microscope (STEM) provides a way to probe the four dimensional phonon band dispersion relation, $\mathrm{S}\left(\mathrm{q}_{\mathrm{x}}, \mathrm{q}_{\mathrm{y}}, \mathrm{q}_{\mathrm{z}}, \omega\right)$, with nanometer spatial resolution [1]. The technique has benefited from significant advances in recent years, including increased efficiency by parallel acquisition using slot-shaped spectrometer entrance apertures and the introduction of low-noise high dynamic range direct detectors for EELS [2]. Together with high-brightness electron sources, brightness-preserving monochromators, and next-generation spectrometers, these improvements have reduced the acquisition time for phonon band dispersion diagrams from hours [3] to minutes [2]. Even with these advances, phonon band structure measurement in the STEM has been limited to ideal systems containing light elements with $Z=5-7$ (e.g. boron nitride, or carbon), where the phonon structure spans roughly 0-200 meV energy loss. Phonon band structures in materials with heavier atoms, where the energy range can be significantly smaller, have been elusive until now.

Figure 1 shows a phonon band dispersion diagram acquired from silicon with probe size $\sim 5 \mathrm{~nm}$, the diffraction limit with $1 \mathrm{mrad}$ illumination half-angle at $30 \mathrm{kV}$. The full width of the pattern is about 65 $\mathrm{meV}$. The transverse acoustic (TA) phonon branch, which rises from the energy origin at the $\Gamma$ point to about $14 \mathrm{meV}$ at the L point, is clearly resolved. The dispersion of the longitudinal acoustic (LA) phonon branch from 0 to $\sim 54 \mathrm{meV}$ is visible. At the L point, the splitting of the longitudinal optical (LO, $\sim 62 \mathrm{meV}$ ) and transverse optical (TO, $\sim 54 \mathrm{meV}$ ) appears as a wide band at this energy resolution. A less intense reflection of the pattern is just visible on the gain side, showing that room temperature $(\mathrm{kT} \sim 25 \mathrm{meV}$ ) thermal excitation of energy gain is significant on this energy scale.

Similar to previous reports [2], the projector lenses were used to rotate and scale the diffraction pattern from the $\sim 70 \mathrm{~nm}$ thick $\mathrm{Si}[110]$ oriented sample such that a rectangular spectrometer entrance aperture , $2 \mathrm{~mm} \times 125 \mathrm{um}$, spanned the [000] and [-111] diffraction spots (i.e the $\Gamma$-L direction in the silicon Brillouin zone). The total acquisition time was $\sim 13 \mathrm{~min}$. The energy resolution in these conditions was about $8 \mathrm{meV}$ full-width-at-half-maximum for the zero loss peak (ZLP), and the current was $\sim 2 \mathrm{pA}$. High angular resolution at high current introduces significant and competing demands on the instrumentation, which tend to degrade the energy resolution. Under typical operating conditions with the Nion UHERMES system used here, $5 \mathrm{meV}$ energy resolution at $30 \mathrm{kV}$ is routine.

Resolving the phonon band dispersion diagram in a variety of materials in the STEM is the first step toward a larger goal of resolving the effect of local structure on vibrational modes [4]. Adding spatial dimensions to the acquisition places additional demands on the system, especially increasing the current so that the signal can be collected in a reasonable time without compromising the energy resolution too 
much to see the pattern. The conceptual and software complexity for acquisition and analysis also increases.

Figure 2 shows a proof-of-principle spatially resolved phonon band dispersion diagram going from silicon into vacuum across a $\sim 5 \mathrm{~nm}$ amorphous layer at the interface. Using the same setup as for Fig. 1 with a wider energy-selecting slit for higher current ( $\sim 8 \mathrm{pA})$ and energy width $(\sim 12 \mathrm{meV})$, the data was acquired as a series of 45 successive line scans of 6 scan pixels each, recording a full 2D pattern on the detector (4s) at each scan point, for a total time of $45 \times 6 \times 4$ s, $\sim 18$ minutes. The data was multiply aligned in post processing to account for slow drift in the energy direction, drift of the sample in space, and to correct for charging of the EELS collection aperture. The result is a 3 dimensional line scan with a 2D phonon band dispersion diagram at each of 6 points in space.

To visualize the 3D data set in Figure 2, two data points are shown: one from the bulk, and one from the amorphous interface with vacuum. Line profiles through the L point are shown on the right in Fig. 2 (i.e. raw data with no normalization). These line plots are similar to the spectrum that would be observed if a round 125um diameter collection aperture had been placed at the $\mathrm{L}$ point. The scattered quasi-ZLP is much more intense at the interface. The TA phonon around $14 \mathrm{meV}$, which appears as a shoulder at this energy resolution, is much stronger in the crystal, as are the LA, TO, and LO phonons. Previous work showed that the dark field phonon signal is as sharp as the probe [5], though the signal right at the interface proved difficult to interpret. Having the whole pattern provides an invaluable resource for clear interpretation of the peaks, and parallel acquisition of the pattern boosts the collection efficiency by a factor of $16 x$, which is critical for higher-dimensional angle and spatial mapping.

In conclusion, the phonon band structure in silicon has been resolved with $<10 \mathrm{meV}$ energy resolution, $\sim 1 \mathrm{mrad}$ angular resolution, and $\sim 5 \mathrm{~nm}$ spatial resolution. We are assembling the tools for optimizing the acquisition as a function of angle and position, including automated multi-frame acquisition with alignment. This will encourage phonon band structure mapping with "in-situ" stimulants such as heat, mechanical stress, or other excitations where vibrational modes and local structure together play a critical role.
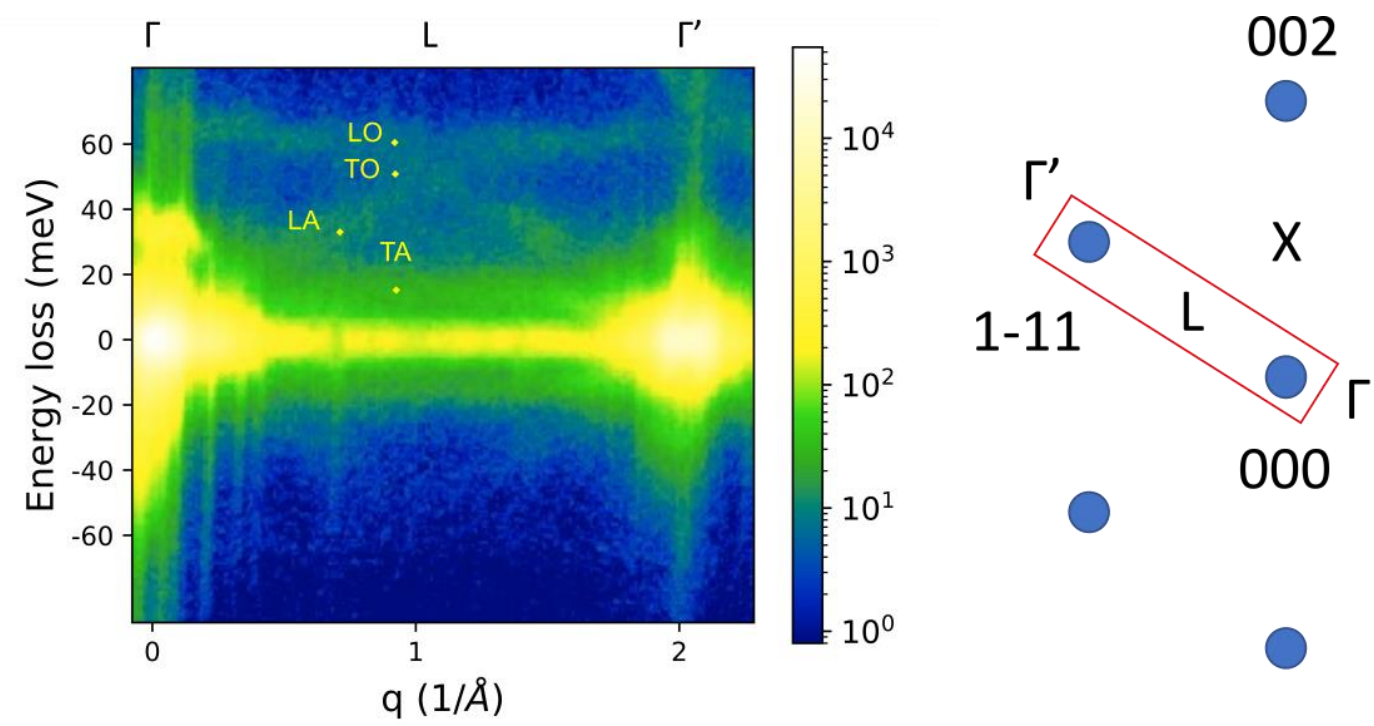
Figure 1. Phonon Band Dispersion Diagram from Silicon. (left) diagram acquired along the $\Gamma$-L direction in the silicon Brillouin zone resolving the transverse acoustic (TA) phonon mode ( $14 \mathrm{meV}$ at the $\mathrm{L}$ point), longitudinal acoustic (LA) modes (0-50 meV) and transverse- and longitudinal-optical (TO and LO, respectively) phonon modes $(\sim 50-60 \mathrm{meV})$. (right) schematic diagram showing the rectangular spectrometer entrance aperture with Brillouin zone labels and diffraction pattern indices. E0 $=30 \mathrm{kV}, \mathrm{Ip}=2$ $\mathrm{pA}, \square / 2=1 \mathrm{mrad}$.
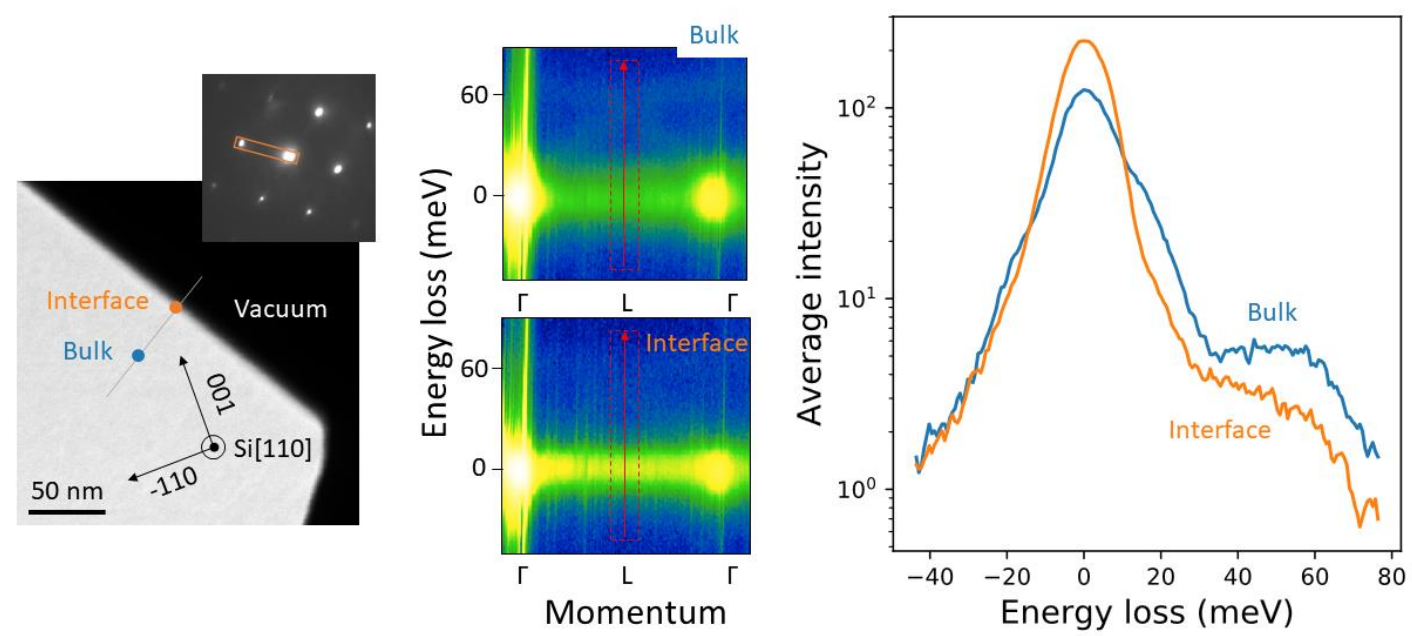

Figure 2. Spatially-resolved phonon band dispersion diagram from Si[110]. (left) Overview of the interface between silicon and vacuum with (inset) diffraction pattern showing the rotation orientation between the interface, diffraction pattern, and the rectangular EELS collection aperture. (upper middle) diagram acquired from the bulk silicon, (lower middle) diagram acquired from the interface. (right) comparison of line profiles through the two diagrams at the L point showing phonon peaks (a shoulder at $14 \mathrm{meV}$, and broad features at 30-60meV) decreasing in intensity at the interface and the quasi-ZLP increasing. Multi-frame acquisition with 45 scans, 6 pixels each, 4s per pixel, 18 min total, multiply aligned. $\mathrm{E} 0=30 \mathrm{kV}, \mathrm{Ip}=2 \mathrm{pA}, \alpha / 2=1 \mathrm{mrad}$.

\section{References}

[1] F.S. Hage et al., Sci. Adv. 2018;4:eaar7495 1-5

[2] B. Plotkin-Swing et al., Ultramicroscopy 217, 113067 (2020).

[3] R. Senga et al., Nature 573, (2019) 247.

[4] R. Qi et al., Nature Comms., (2021) doi.org/10.1038/s41467-021-21452-5

[5] C. Dwyer et al., Phys. Rev. Lett. 117 (2016) 256101 